\title{
PHOSPHOTENSIN TUMOUR SUPPRESSOR GENE (PTEN) EXPRESSION PATTERNS IN ENDOMETRIAL HYPERPLASIAS AND ENDOMETRIOID CARCINOMA
}

\author{
Sithara $S^{1}$, Sheela Varghese2, Sankar $S^{3}$
}

${ }_{1}^{1}$ Resident, Department of Pathology, Government Medical College, Kottayam, Kerala, India.

${ }^{2}$ Additional Professor, Department of Pathology, Government Medical College, Kottayam, Kerala, India.

3 Professor and HOD, Department of Pathology, Government Medical College, Kottayam, Kerala, India.

\begin{abstract}
BACKGROUND

Endometrioid Endometrial Carcinoma (EECA) has been postulated to develop from premalignant lesions like endometrial hyperplasia without atypia, to endometrial hyperplasia with atypia. Various studies have shown PTEN tumour suppressor gene mutation in endometrioid carcinoma and its precursors. Thus, immunohistochemical expression of PTEN may provide a valuable insight into the category of hyperplasia which can be most definitely classified as "premalignant". This study evaluated PTEN expression in endometrial hyperplasia with and without atypia and endometrioid carcinoma.
\end{abstract}

\section{MATERIALS AND METHODS}

The present study evaluated PTEN expression in endometrial hyperplasia with and without atypia and endometrioid carcinoma in endometrial curetting and hysterectomy specimens received in Government Medical College, Kottayam from 18/4/2017- 9/9/18.

\section{RESULTS}

The mean age of study population was $56.98 \pm 10.67$ years. PTEN is strongly immunoreactive in all cases of proliferative and secretory phase. There was $100 \%$ positivity or $0 \%$ negativity in endometrial hyperplasia without atypia. In $37 \%$ cases of endometrial hyperplasia with atypia and $61 \%$ cases of endometrioid carcinoma, there was complete loss of PTEN expression.

\section{CONCLUSION}

Loss of PTEN expression is partially associated with endometrioid endometrial cancers via a premalignant phase.

\section{KEY WORDS}

PTEN (Phosphotensin Tumour Suppressor Gene); Endometrial Hyperplasia; Endometrioid Carcinoma; Immunohistochemistry.

HOW TO CITE THIS ARTICLE: Sithara S, Varghese S, Sankar S. Phosphotensin tumour suppressor gene (PTEN) expression patterns in endometrial hyperplasias and endometrioid carcinoma. J. Evolution Med. Dent. Sci. 2019;8(07):403-406, D0I: 10.14260/jemds/2019/89

\section{BACKGROUND}

Endometrial cancer is the fourth most common cancer of women in developed countries and most common amongst the cancers of the female reproductive tract. ${ }^{1}$ Endometrial carcinoma ranks third in India among gynaecological malignancies. ${ }^{2}$ Endometrial hyperplasias have been widely regarded as the associated precursor lesion. ${ }^{3}$ Endometrioid endometrial carcinoma (EECA) accounts for three fourths of endometrial cancers and are believed to develop following a continuum of premalignant lesions ranging from endometrial hyperplasia without atypia, to endometrial hyperplasia with atypia and finally to well differentiated carcinoma.4,5 It is of importance for the pathologist to identify the subset or factors which could be a possible forerunner of malignancy, especially in hyperplasias. Of the numerous genes involved, the phosphotensin tumour suppressor gene (PTEN) has been identified as the most commonly mutated one in endometrial pre-cancers and cancer.

'Financial or Other Competing Interest': None.

Submission 21-12-2018, Peer Review 31-01-2019,

Acceptance 07-02-2019, Published 18-02-2019.

Corresponding Author:

Sithara S,

Resident,

Department of Pathology,

Government Medical College,

Kottayam, Kerala, India.

E-mail: sitharashoukkath@gmail.com

DOI: $10.14260 /$ jemds/2019/89

It is being extensively researched upon as an informative marker for delimiting neoplastic and pre-neoplastic glands from normal background endometrial glands. The utility of PTEN expression as a marker for atypical hyperplasias progressing to malignancy could perhaps help provide valuable insight into the category of hyperplasias which can be most definitely classified as "premalignant". This study evaluates PTEN expression in endometrial hyperplasia and endometrioid carcinoma.

\section{MATERIALS AND METHODS}

\section{Type of Study}

Descriptive study.

\section{Period of Study}

18 months (18/4/2017- 9/9/18)

\section{Study Setting}

Department of Pathology, Government Medical College, Kottayam.

\section{Study Population}

Endometrial biopsies and hysterectomy specimens with histopathological features diagnostic of endometrial hyperplasia and endometrioid endometrial carcinoma received in the Department of Pathology, Government medical college, Kottayam. 


\section{Sample Size}

Calculated by the formula $\mathrm{z}^{2} \mathrm{pq} / \mathrm{d}^{2}$

Where $\mathrm{p}=$ prevalence $/$ proportion of cases with PTEN loss in the parent study

$\mathrm{q}=100-\mathrm{p}$

$\mathrm{d}=$ precision (Allowable error)

Here, $p=60$ (From study conducted by Patou Tantbirojn et al. $)^{6}$

$\mathrm{q}=40$

$\mathrm{d}=20 \%$ of $\mathrm{p}, \mathrm{z}=1.96$ at $95 \%$ confidence interval.

\section{Study Procedure}

- All specimens are received in formalin. These are processed, paraffin embedded in total. Thin (5 Micrometer) sections were taken and stained by haematoxylin and eosin and IHC study for PTEN was done by Standard Operating Procedure. PTEN intensity and scoring was done according to Soheila Sarmadi et al. ${ }^{7}$ and Tantbirojn ${ }^{6}$ et al.

- According to majority of studies, immunoreactivity was regarded as positive when brown staining was localized in the nuclei or cytoplasm of normal endometrial gland cell or tumour cell. According to Kapucuoglu et al $^{15}$ and Sarmadi et al ${ }^{7}$ the immunoreactivity was graded semi quantitatively by considering the percentage and intensity of staining on the whole section. Staining of cells was scored as negative if $<10 \%,+1$ if $10 \%-50 \%$ and +2 if $>50 \%$ of slide's area was stained positive. The intensity of PTEN staining was scored from $0=$ absent, $+1=$ light brown, $+2=$ brown to dark brown in the nucleus or cytoplasm of glandular cells for each specimen. $6,7,14,15$

\section{Data Management and Analysis}

The data entered in Microsoft excel was statistically analysed using SPSS 24.

\section{Mean, Frequency and Proportion For}

1. Age.

2. Histopathology of endometrial lesions studied.

3. PTEN scoring and intensity in various endometrial lesions.

4. Relation between PTEN scoring and intensity with endometrial hyperplasias with and without atypia and endometrioid carcinoma using chi square test ( $p$ value).

\section{RESULTS}

7 specimens of endometrial hyperplasia without atypia, 8 specimens of endometrial hyperplasia with atypia and 49 specimens of endometrioid carcinoma were studied.

The mean ages of patients with endometrial hyperplasias without and with atypia and endometrioid carcinomas were $54.43 \pm 5.99$ years, $54.75 \pm 17.91$ years and $58.02 \pm 9.48$ years respectively.

- PTEN was strongly immunoreactive in all cases of proliferative and secretory phase in the current study.

- There was $100 \%$ positivity or $0 \%$ negativity in endometrial hyperplasia without atypia.

- There was complete PTEN loss (Negative immunoreactivity) in $37 \%$ cases of endometrial hyperplasia with atypia and $61 \%$ cases of endometrioid carcinoma.

- $\quad$ PTEN immunoreactivity between the different groups of endometrial hyperplasias and endometrioid carcinoma was statistically significant $(\mathrm{p}<0.001)$.

- Difference in immunoreactivity between endometrial hyperplasia with atypia and endometrioid carcinoma was not statistically significant.

\section{Gross and Microscopy}
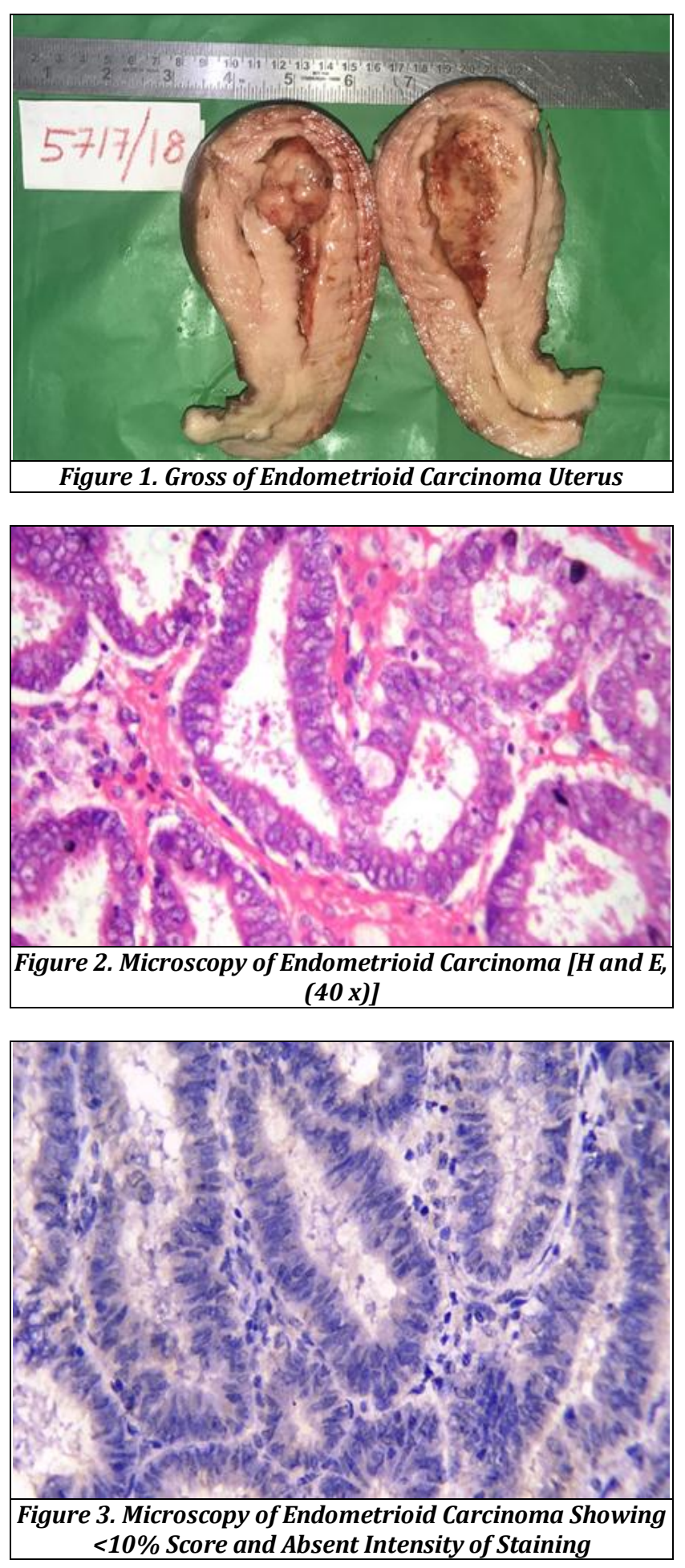

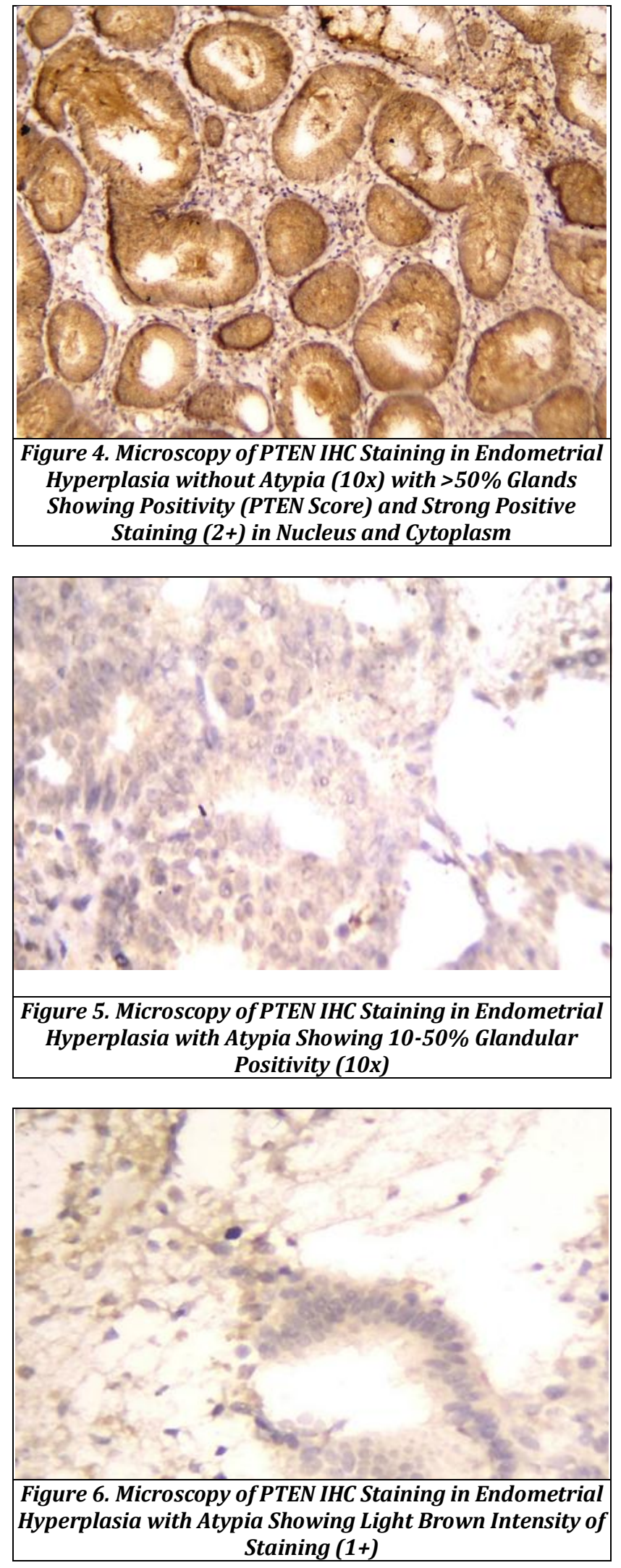

\section{DISCUSSION}

Endometrial cancer is common in western women with very high rates; but in India, the incidence rates are as low as 2.8 per 100, 000 (Mumbai). ${ }^{8}$ Hence the studies on endometrial cancer in India are limited. The term "endometrial neoplasia" is a spectrum of morphologic alterations which range from endometrial hyperplasia to endometrial carcinoma.(9)
PTEN, a tumour-suppressor gene, mutates in $30-50 \%$ of endometrioid carcinomas, a rate that is among the highest of any type of tumour analysed to date. It is also found to be mutated in about $13-55 \%$ of endometrial hyperplasias. 10 Moreover, PTEN mutation was seen favourable endometrial carcinoma with a favourable survival.11

This study evaluated the differential expression of PTEN in endometrial hyperplasia and endometrioid carcinoma. This study was done on 7 cases of endometrial hyperplasia without atypia, 8 cases of endometrial hyperplasia with atypia and 49 cases of endometrioid carcinoma. Positive control taken was that of proliferative and secretory endometrium.

\section{Comparison of PTEN Loss}

\begin{tabular}{|c|c|c|c|c|}
\hline $\begin{array}{l}\vec{E} \\
\stackrel{\vec{n}}{*}\end{array}$ & 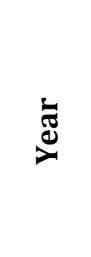 & 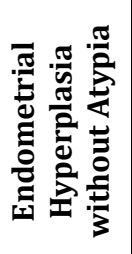 & 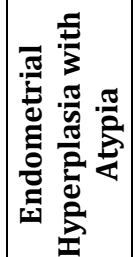 & 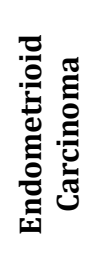 \\
\hline Soheila Sarmadi et.al7 & 2009 & $0 \%$ & $25 \%$ & $52 \%$ \\
\hline Shanmugapriya et.al 14 & 2017 & $11 \%$ & $50 \%$ & $70 \%$ \\
\hline Tantbirojn et. al ${ }^{6}$ & 2008 & $24 \%$ & $60 \%$ & $60 \%$ \\
\hline Present Study & 2018 & $0 \%$ & $37 \%$ & $61 \%$ \\
\hline
\end{tabular}

PTEN is strongly immunoreactive in all cases of proliferative and secretory phase in the current study. These findings agreed with Soheila Sarmadi et al (2009), in which PTEN immunoreactivity was noted in all proliferative endometrium (29/29, 100\%). PTEN expression was significantly higher in proliferative and secretory endometrium than in endometrial hyperplasia with atypia and endometrioid carcinoma.

In endometrial hyperplasia, loss of PTEN expression is found to be $30-63 \% .^{(12,13,14)}$ Mutter et al. detected loss of PTEN expression in $55 \%$ and $63 \%$ in endometrial intraepithelial neoplasias in two different studies.(13)

The current study showed $100 \%$ positivity or $0 \%$ negativity in endometrial hyperplasia without atypia which is similar to the study by Soheila Sarmadi et al (2009). Studies conducted by Shanmugapriya et al (2017) and Tantbirojn et al (2008) gave comparable findings, i.e, only $11 \%$ and $24 \%$ cases of endometrial hyperplasia without atypia were negative for PTEN expression.

Our study showed complete PTEN loss or negative immunoreactivity in $37 \%$ cases of endometrial hyperplasia with atypia, which is almost comparable to PTEN loss in studies conducted by Soheila Sarmadi et al and Shanmugapriya et. al.; which were $25 \%$ and $50 \%$ respectively. Study conducted by Tantbirojn et al showed PTEN negativity in a greater proportion of about $60 \%$ cases. Kapucuoglu et al. found complete loss of PTEN in $20 \%$ of atypical complex samples. 15

The present study showed PTEN negative immunoreactivity in $61 \%$ cases of endometrioid carcinoma which is similar to the findings of Tantbirojn et al (60\%) and comparable to the findings of Sohelia Sarmadi et al (52\%) 
and Shanmugapriya et.al. (70\%). Orbo et al reported loss of PTEN protein expression in 55\% of specimens in patients who developed subsequent endometrioid endometrial carcinoma. ${ }^{16}$

Latta E et al (2002) found mutations of the PTEN tumour suppressor gene are present in histologically normalappearing endometrium exposed to oestrogen, $18-55 \%$ of endometrial precancers and $26-80 \%$ of EECs. ${ }^{17}$

There was significant difference in PTEN immunoreactivity between different endometrial hyperplasia and endometrioid carcinoma. $(\mathrm{p}<0.001)$. But, there was no significant difference in immunoreactivity between endometrial hyperplasia with atypia and endometrioid carcinoma.

\section{CONCLUSION}

PTEN expression in endometrioid carcinoma and its precursors was as follows-

- PTEN showed a positive expression in $100 \%$ cases of endometrial hyperplasia without atypia.

- Endometrial hyperplasia with atypia showed complete loss of PTEN or negative immunoreactivity in $37 \%$ cases.

- $\quad$ PTEN showed negative immunoreactivity in $61 \%$ cases of endometrioid carcinoma.

- Thus, from our study, diminished PTEN expression was associated with malignant endometrium with statistically significant difference in PTEN immunoreactivity between normal endometrium, hyperplasias and carcinoma. $(\mathrm{p}<0.001)$

Our data suggested that loss of PTEN expression is partially associated with the endometrioid carcinomas via a premalignant phase.

\section{ACKNOWLEDGEMENTS}

I express my heartfelt thanks and gratitude to Dr. Sankar S, Head of the Department, Department of Pathology, Government Medical College, Kottayam and my guide Dr. Sheela Varghese for their support and help. I am thankful to all my teachers in the Department of Pathology as well as fellow post graduate students and my friends for their wholehearted co-operation and encouragement.

\section{REFERENCES}

[1] Jemal A, Siegel R, Ward E, et al. Cancer statistics, 2008. CA Cancer J Clin 2008;58(2):71-96.

[2] Devi UK. Current status of gynecological cancer care in India. Journal of Gynecologic Oncology 2009;20(2):7780.

[3] Creasman WT. Endometrial cancer: incidence, prognostic factors, diagnosis and treatment. Semin Oncol 1997;24(1 Suppl 1):S1-140, S1-50.

[4] Kendall B, Ronnett B, Isacson C, et al. Reproducibility of the diagnosis of endometrial hyperplasia, atypical hyperplasia, and well-differentiated carcinoma. The American Journal of Surgical Pathology 1998;22(8):1012-9.
[5] Mutter GL. Diagnosis of premalignant endometrial disease. Journal of Clinical Pathology 2002;55(5):32631.

[6] Tantbirojn P, Triratanachat S, Trivijitsilp $\mathrm{P}$, et al. Detection of PTEN immunoreactivity in endometrial hyperplasia and adenocarcinoma. J Med Assoc Thai 2008;91(8):1161-5.

[7] Sarmadi S, Izadi-Mood N, Sotoudeh K, et al. Altered PTEN expression: a diagnostic marker for differentiating normal, hyperplastic and neoplastic endometrium. Diagnostic Pathology 2009;4(1):41.

[8] Balasubramaniam G, Sushama S, Rasika B, et al. Hospital-based study of endometrial cancer survival in Mumbai, India. Asian Pacific Journal of Cancer Prevention 2013;14(2):977-80.

[9] Terakawa N, Kanamori Y, Yoshida S. Loss of PTEN expression followed by Akt phosphorylation is a poor prognostic factor for patients with endometrial cancer. Endocrine Related Cancer 2003;10(2):203-8.

[10] Risinger JI, Hayes AK, Berchuck A, et al. PTEN/MMAC1 mutations in endometrial cancers. Cancer Res 1997;57(21):4736-8.

[11] Akiyama-Abe A, Minaguchi T, Nakamura Y, et al. Loss of PTEN expression is an independent predictor of favourable survival in endometrial carcinomas. British Journal of Cancer 2013;109(6):1703-10.

[12] An HJ, Lee $\mathrm{YH}$, Cho NH, et al. Alteration of PTEN expression in endometrial carcinoma is associated with down-regulation of cyclin-dependent kinase inhibitor, p27. Histopathology 2002;41(5):437-45.

[13] Mutter GL, Ince TA, Baak JP, et al. Molecular identification of latent precancers in histologically normal endometrium. Cancer Res 2001;61(11):43114.

[14] Shanmugapriya M, Sudha M, Geetha P. A study of PTEN expression in endometrial hyperplasia and endometrioid type of endometrial carcinoma. Trop J Path Micro 2017;3(1):39-45.

[15] Kapucuoglu N, Aktepe F, Kaya H, et al. Immunohistochemical expression of PTEN in normal, hyperplastic and malignant endometrium and its correlation with hormone receptors, bcl-2, bax and apoptotic index. Pathology - Research and Practice 2007;203(3):153-62.

[16] Orbo A, Kaino T, Arnes M, et al. Genetic derangements in the tumor suppressor gene PTEN in endometrial precancers as prognostic markers for cancer development: a population-based study from northern Norway with long-term follow-up. Gynecol Oncol 2004;95(1):82-8.

[17] Latta E, Chapman WB. PTEN mutations and evolving concepts in endometrial neoplasia. Curr Opin Obstet Gynecol 2002;14(1):59-65. 